\title{
Bioanalysis
}

\section{Giving consideration to scientific validation in Japanese drug application by Japan Bioanalysis Forum discussion group}

\author{
"Scientific validation is still a new concept in Japan, hence, the first \\ purpose of our activity is to have it well-recognized and discussed in \\ detail."
}

First draft submitted: 21 April 2017; Accepted for publication: 8 May 2017;

Published online: 13 July 2017

Keywords: Bioanalytical Method Validation $\bullet$ BMV $\bullet$ guideline $\bullet$ drug metabolites analysis - early clinical development $\bullet$ early preclinical development $\bullet$ EBF $\bullet$ European Bioanalysis Forum • JBF • Japan Bioanalysis Forum • scientific validation • SV • tiered approach • tissue analysis $\bullet$ urine analysis

\section{Background}

Scientific validation (SV) is a concept proposed by the European Bioanalysis Forum (EBF) and it utilizes both scientific discretion and validation in characterizing analytical methods. SV could improve drug-development efficiency as a component of a tiered approach by streamlining the process of analytical method establishment and validation. The EBF has summarized and published SV as a White Paper [1]. In the White Paper, a total of five categories are listed as assay-appropriate SV constituting three categories (metabolites in plasma for assessment of ICH-M3(R2) criteria, unchanged form in urine and tissue homogenates) and stage-appropriate SV constituting two categories (unchanged form in plasma at early stage clinical studies and preclinical studies). There are many discussions about bioanalytical validation parameters in each category.

The Japan Bioanalysis Forum (JBF) was established in 2011 [2]. As one of its activities, voluntary discussion groups (DGs) have been organized since 2013. Each DG selects and discusses a bioanalytics theme, and presents it at the JBF symposium. In 2015, DG2015-16 selected SV as a main activity theme. First, the DG members shared and discussed the contents of the white paper in detail applicable to the Japanese New Drug Application (NDA). Then the results of discussions were summarized and presented in the form of a poster at the 2016 JBF symposium [3]. Based on the results of the poster presentation, DG201620, which is a new DG for the 2016 fiscal year, performed a survey to collect opinions from the Japanese bioanalytical community (JBF DG supporters and their colleagues).

In this article, we describe our discussion and survey results regarding SV points applicable to Japanese NDA submission.

\section{Survey on SV}

Initially, the DG held internal discussion to investigate whether characterization items and validation systems of SV proposed by the EBF were scientifically defendable. SV items and systems, which was divided into each of the five categories mentioned above, were also confirmed for their acceptability to regional practices. From every viewpoint, SV items and systems were considered to be appropriate.

As a next step, the general acceptance of typical characterization items in the White Paper was surveyed. The survey was done using the web tool for the JBF DG supporters who are mailing list members having
Nozomu Koseki*,1, Hiroko Ashizawa², Takuho Ishii ${ }^{3}$, Takahide Uchimura ${ }^{4}$, Keita Ono 5 , Saori Kuriyama ${ }^{6}$, Akiko Toda7, Kuretake Soejima ${ }^{8}$, Naoko Nakai ${ }^{9}$, Naohiro Nishimura ${ }^{10}$, Tsuyoshi Mayumi11, Yutaka Yasuda'2, Tatsuya Yamakawa ${ }^{13}$ \& Makoto Niwa ${ }^{14}$

*Author for correspondence: Tel.: +81 28057 1551; nozomu.koseki@mb.kyorin-pharm.co.jp

For author affiliations, please see the end of this article.

newlands press part of 
interest in DG activities organized by the JBF, and are composed of Japanese bioanalysts with a broad range of background and experience. Responses were obtained from 58 individuals, which was considered as an appropriate representation of the trend among Japanese bioanalysts.

\section{The results of the survey $\&$ discussion}

The SV survey contained the following aspects:

- The use of the certificate of analysis (COA) ICHM3(R2) (investigation or determination of metabolite exposure to see the necessity of metabolite safety testing);

- Pros/cons for Interassay accuracy and precision (A\&P; Interassay A\&P is not mandatory);

- Scientific justification of using internal standard (IS) variability for detecting matrix effects and hemolysis impact;

- Possible abbreviation of stability testing;

- Opinions on NDA filing of the data obtained by SV.

The followings are the main discussion points on SV from the White Paper, DG and the survey.

\section{Background of respondents}

The major proportion of organizational affiliation is with domestic pharmaceutical companies at $74 \%$, domestic contract research organizations (CROs) at $21 \%$ and foreign pharmaceutical companies at only $3 \%$. Since foreign pharmaceutical companies do not have laboratories in Japan, they may not be interested in SV. Respondents' ages and duration of bioanalytical experience cover a wide range.

\section{COA}

The EBF recommendation was to require a COA or analysis using dosed batch for unchanged form, how-

"The European Bioanalysis Forum
recommendation was that stock solution
stability should be investigated in early method
development for analysis of unchanged form."

ever no requirement for metabolites. The DG opinion was to require at least result of analysis (ROA [reports key quality items such as purity, but does not fulfill the requirement of the Bioanalytical Method Validation (BMV) guideline for analysis of unchanged form]), and to require an ROA in exposure assessments if the metabolites should be further evaluated for safety based on ICH-M3(R2). An agreement from the JBF DG supporters in 'tiered approach' [4] was that a metabolite COA for quantitative determination is not required but an $\mathrm{ROA}$ is.

About $80 \%$ of respondents agreed with the DG's opinion. However, the definition of ROA is not commonly defined yet.

\section{Number of points for the calibration curve}

The EBF recommendation for the number of points for the calibration curve was at least five points for metabolites, urine and tissues, and six points for early development in plasma. The DG agreed with the EBF, however, the number of points should be increased in the case of a wide analytical range.

Most of the respondents agreed to either the DG's or EBF's opinion. The DG and EBF opinions seemed to be no different in practical terms.

\section{Criteria of calibration curve \& QC}

The DG agreed with the EBF proposal, which was $20 / 25 \%$ criteria for early development in plasma and $25 / 30 \%$ for metabolites, urine and tissues, and added one regional aspect that urine analysis for mass balance should be controlled under more stringent criteria.

Although, about half of the respondents selected $20 / 25 \%$ in any measurement, in early clinical development for analysis of unchanged form, there was a preference to $15 / 20 \%$ criteria. Relaxed criteria for analysis of unchanged form in an early clinical study were not accepted, however, accepted for metabolite and tissue concentration measurement. In general, there is an opinion that the criteria should be determined on a case-by-case basis.

\section{Levels/number of replicates of QC sample}

The EBF proposed three concentrations/three replicates of QC samples for metabolites, urine, and tissues, and four concentrations/five replicates for plasma in early studies in pre-study validation. The DG added one regional aspect, that LLOQ can be considered when analysis near the LLOQ is significant. Approximately $2 / 3$ of the respondents agreed with the DG opinion, the other $1 / 3$ agreed with the EBF recommendation.

\section{Interassay variability}

The EBF recommended intraday variability to be confirmed in a pre-study validation. The DG added the viewpoint that the results of $\mathrm{QC}$ samples in instudy validation can be used as retrospective interday variability.

Half of the respondents (47\%) agreed with the EBF recommendation and $40 \%$ agreed to the DG's proposal. As for the evaluation method for retrospective 
interday variability, $58 \%$ of the respondents answered that the variability within the measurement batch was confirmed by the acceptance frequency $4 / 6$ rule. The other $42 \%$ answered that intraday accuracy and precision should be retrospectively evaluated by all QC results in the study.

\section{Selectivity}

The EBF recommendation was six individual samples for analysis of unchanged form in plasma in the early clinical stage. For the others, selectivity could be confirmed by a single sample. The DG agreed with this recommendation.

In the survey, the major answer for analysis of unchanged form in the early clinical stage was six samples. Various responses for other items were obtained, but the use of single pooled matrix was the majority answer.

Selectivity in the presence of co-medication in drug-drug interaction studies was also surveyed. Over $80 \%$ of respondents answered that they had not experienced that drug-drug interaction affected the selectivity while $17 \%$ of respondents had. Major issues were abnormal chromatograms by interference.

\section{Stability of standard stock solutions}

The EBF recommendation was that stock solution stability should be investigated in early method development for analysis of unchanged form. Unless stability is investigated, stock solution should be prepared just before use. For analysis of metabolites, minimal assessment is enough and the recommended criteria were $100 \pm 20 \%$. In DG discussion, stability evaluation was generally considered necessary.

Three quarters $(76 \%)$ of the respondents stated that stability investigation was necessary. There were some opinions that stability was more important for some unstable metabolites. Stability criteria setting was difficult in the case of labile metabolites and it was dependent on the nature of the analytes.

\section{Freeze-thaw stability}

The EBF recommended various approaches for freeze-thaw $(\mathrm{F} / \mathrm{T})$ stability. For early phase studies, F/T stability should be performed in conjunction with other stability assessments. For analysis of metabolites, F/T stability could be covered with incurred sample stability (ISS). For urine and tissue samples, one cycle F/T stability should be assessed. In the DG, it was considered that generally all-at-once stability testing could be recommended. There were some opinions saying that ISS was not enough for some situations because ISS results are retrospective.

Most of respondents $(80 \%)$ agreed with the DG. Some respondents stated that ISS was acceptable because it was good enough if stability was ensured at the end of the study.

\section{Long-term storage stability}

The EBF recommended long-term storage stability should be performed in conjunction with other stability assessments with relaxed criteria $(100 \pm 20 \%)$ for analysis of metabolites. For urine and tissue, long-term storage stability was not necessary. In the DG, it should be assessed based on the purpose of the experiment.

In the survey, over $80 \%$ of respondents agreed with the DG; stability in urine and tissue is to be assessed, if necessary.

\section{Whole blood stability}

Although, the EBF discussed whole blood stability testing [5], recommendation in SV was unnecessary evaluation [1] and the DG agreed, unless there

"Simplification of the protocol and report is recommended by the European Bioanalysis Forum and the discussion group also agreed."

is specific chemical structure indicating instability in whole blood. The EBF BMV guideline [6] has also not reached agreement in the necessity of whole blood stability testing and is still discussing it in, for example, an open discussion session with regulators in the 7 th JBF symposium [3].

Most of the respondents (95\%) stated that whole blood stability testing is unnecessary. A few of respondents stated that stability should be evaluated, if necessary.

\section{Bench-top \& processed sample}

In the early stage for analysis of unchanged form, the EBF and DG recommendation concurred on the points that bench-top stability should be performed in conjunction with other stability assessments, processed sample stability should be assessed based on scientific judgment. On the other hand, for metabolites, urine and tissue, it is unnecessary. Survey results indicated more than $80 \%$ of the respondents stated that they agree with the EBF and DG's opinion.

\section{Study results evaluation by IS variability}

More than $80 \%$ of respondents did not apply IS variability. Although, several respondents attempted IS variability; the criteria of IS variability are unclear. Among the responses stating that they employ IS variability criteria, the criteria of $50 \%$ are often used. The EBF recommendation [7] is not commonly applied yet, but it is acceptable. Several respondents commented that the criteria should be changed between stable isotope-labeled (SIL)-IS and analog-IS for IS variability. 
Evaluation of hyperlipidemic/hemolysis samples by IS variability

The EBF proposed that effects of hyperlipidemic or hemolysis plasma samples could be evaluated by IS variability in in-study validation. The DG agreed, although it recognized the limitation that the impact of hemolysis of high erythrocyte-binding drugs could not be assessed by IS variability.

About $60 \%$ of the respondents agreed with this approach. Many opinions obtained indicated that a different approach should be taken based on the nature of IS (SIL or analog).

\section{Carry over}

The EBF and DG's opinion were that carry over can be assessed in in-study validation. The DG also considered when carry over was detected during analytical method development, corrective action should be required, but if not, it was just assessed in in-study validation. Many respondents answered agreeing with the opinion of the DG.

\section{ISR}

The EBF proposed incurred sample reanalysis (ISR) was not required for metabolite, urine and tissue analysis. The DG's opinion, however, was appropriate action based on the significance, such as pharmacological impact. Over $90 \%$ of respondents would not require or do it unless necessary.

\section{Dilution integrity}

Dilution integrity test was required in the case of analysis for dilution sample(s) in in-study validation. The DG also required a pre-study validation in a predictable case, such as the Single Ascending Dose study. Respondents answered, if necessary, a dilution integrity test was required in pre-study validation $(2 / 3)$ and in-study validation (1/3) depending on the case.

\section{Sampling method}

The EBF emphasized the importance of preliminary investigation of the sampling method (avoiding adsorption or stabilization) and documentation. The DG agreed with the EBF, and survey results indicated about $80 \%$ of respondents agreed with the EBF and DG.

In the DG discussion, it was important how documentation was prepared.

\section{Extrapolation of calibration range}

According to the EBF, extrapolation over the calibration range within an admitted allowance of error (i.e., $+20 \%$ of the ULOQ or $-25 \%$ of the LLOQ) is acceptable, as long as it is scientifically justified (linear model and sufficient $\mathrm{S} / \mathrm{N}$ ). The DG agreed with the
EBF, however, in the case of the ULOQ, it is appropriate to re-assay after sample dilution, if accurate results are required.

In the case of ULOQ, half of the respondents agreed to extrapolation within an accuracy criterion $(+20 \%)$. However, one third of respondents answered that re-assay is necessary. In the case of LLOQ, half of the respondents did not accept extrapolation.

\section{Re-analysis of anomalous results (including positive control or predose sample)}

The EBF recommendation was anomalous results reanalysis should not be executed for metabolite, urine and tissue, but should be executed for plasma in duplicate. The DG discussion reached the consensus that re-analysis should be based on scientific judgment for metabolite, urine and tissue, and should be performed based on the SOPs or protocol for plasma.

In the survey, $75 \%$ of respondents executed re-analysis by scientific judgment for metabolite, urine and tissue. In the case of analysis of unchanged form in plasma, $80 \%$ of the respondents re-analyzed based on the SOPs or protocol.

\section{Protocol, report \& SOP}

Simplification of the protocol and report is recommended by the EBF and the DG also agreed. Three quarters of respondents agreed with the EBF and DG. Over $90 \%$ of respondents were not prepared for SOPs for SV or tiered approach (TA). However, over $40 \%$ of respondents have experience with SV or TA even though they do not have SOPs.

\section{NDA by SV}

As there is an opinion among some stakeholders that SV data (even for insignificant metabolites) are not appropriate for NDA in any circumstances because it is not BMV guideline-compliant, the understanding among the bioanalysts was surveyed. The final question of the survey questioned the possibility of the acceptance of the five categories in SV by the Japanese NDA:

- Analysis of metabolite in plasma: those who believed that NDA by SV is impossible were in minority $(15 \%)$;

- Analysis of unchanged form in urine: the percentage of respondents who think NDA is impossible was higher than the case of metabolite in plasma (30\%);

- Analysis of unchanged form in tissue (homogenate): results were similar to the response for metabolite in plasma (impossible: 15\%); 
- Analysis of unchanged form in plasma in the early nonclinical development stage: the opinion that full validation is required was $30 \%$. There were some opinions that they do not count on SV in supporting NDA, because toxicokinetic studies under GLP are conducted without any exceptions and this will fulfill information requirements on animal studies at the time of NDA.

- Analysis of unchanged form in plasma in the early clinical development stage: many (60\%) respondents considered that application by SV could not be approved, because a clinical study needs to be within the main scope of the BMV guideline. There was an opinion that SV was acceptable for a micro-dose study.

\section{Discussion \& conclusion}

Based on the DG internal discussion on SV, which is a concept proposed by the EBF [1], a web tool survey was performed to JBF DG supporters who were mailing list members. Responses were obtained from 58 individuals.

Our survey intended to help practical implementation of SV by showing various information related to this idea. As a result, the survey showed current practical implementation of SV, and trends in utilizing the data for NDA filing in Japan. This result was appropriate for the purpose of observing the trend among Japanese bioanalysts.

SV is generally accepted in Japan, which is an EBF opinion that does not always comply work in parallel with the BMV guidelines. There are no obvious evaluation standards for urine, tissue or metabolite in plasma analysis, which are not in the main scope of the BMV guideline, and categorized as assay-appropriate SV by the EBF [1].

With regard to metabolite in plasma, a TA was discussed in the Global Bioanalysis Consortium [8] as well as the EBF [1,9], and described in the MHLW BMV guideline [10], and our previous DG (DG201409) also discussed and published it [4], however urine and tissue has been less discussed. Assay-appropriate SV may provide basic ideas for evaluation in these areas.

On the other hand, stage-appropriate SV, which is an analysis of early development of preclinical and clinical studies as a part of a TA in the drug-development stage, was not overly expected by respondents to be acceptable for current Japanese NDA filing. Although stage-appropriate SV is useful for rapid development of a new chemical entity, some bioanalysts do not want to use it for early phase clinical studies, because they think these data are important in NDA. It may drive them to perform bioanalysis of early phase studies in accordance with the BMVguideline. Thus the importance of recognizing the use of the data is highlighted.

SV/TA is actually applied in some form in Japan, as typically shown by the survey results where $40 \%$ of respondents have experience in TA or SV. SV is still a new concept in Japan, hence, the first purpose of our activity is to have it well-recognized and discussed in detail. Then, SV will be utilized as an effective tool for NDA without over-qualification of the data.

\section{Acknowledgements}

The authors thank the steering committee of the Japanese Bioanalysis Forum for providing authors the chance to discuss the items of interest and to present the results of discussion. The authors also thank Japanese Bioanalysis Forum partners for their participation in the survey and attendees of the 7th and 8th Japanese Bioanalysis Forum symposium for discussion during the poster session at the symposium.

\section{Disclaimer}

The discussion and conclusions are the result of voluntary discussion within the discussion group and are not the authorized opinion of the JBF or Japanese regulatory authorities.

\section{Financial \& competing interests disclosure}

N Koseki is an employee of KYORIN Pharmaceutical Co., Ltd. $\mathrm{H}$ Ashizawa is an employee of Kaken Pharmaceutical Co., Ltd. T Ishii is an employee of Sunplanet Co., Ltd. T Uchimura is an employee of Chugai Research Institute for Medical Science Inc. K Ono is an employee of Taiho Pharmaceutical Co., Ltd. S Kuriyama is an employee of Nippon Shinyaku Co., Ltd. A Toda is an employee of Shin Nippon Biomedical Laboratories, Ltd. K Soejima is an employee of Meiji Seika Pharma Co., Ltd. N Nakai is an employee of Daiichi Sankyo Co., Ltd. N Nishimura is an employee of Sumika Chemical Analysis Service, Ltd. T Mayumi is an employee of Zensei Pharmaceutical Co., Ltd. Y Yasuda is an employee of Toray Research Center, Inc. T Yamakawa is an employee of Fujifilm Corporation. M Niwa is an employee of Nippon Kayaku Co., Ltd. The authors have no other relevant affiliations or financial involvement with any organization or entity with a financial interest in or financial conflict with the subject matter or materials discussed in the manuscript apart from those disclosed.

No writing assistance was utilized in the production of this manuscript.

\section{Open access}

This work is licensed under the Attribution-NonCommercialNoDerivatives 4.0 Unported License. To view a copy of this license, visit http://creativecommons.org/licenses/by-nc-nd/4.0/ 


\section{References}

1 Timmerman P, White S, McDougall S et al. Tiered approach into practice: scientific validation for chromatography-based assays in early development - a recommendation from the European Bioanalysis Forum. Bioanalysis 7(18), 2387-2398 (2015).

2 Katori N. Regulated bioanalysis in Japan: where do we come from and where are we going? Bioanalysis 5(11), 1321-1323 (2013).

3 Nakamura T. 7th Japan Bioanalysis Forum symposium: regulated bioanalysis, to a new stage. Bioanalysis 8(20), 2097-2102 (2016).

4 Niwa M, Koseki N, Hashimoto Y et al. Survey on the tiered approach for Japanese bioanalysts operated by Japan bioanalysis forum DG2014-09. Bioanalysis 8(2), 93-98 (2016).

5 Freisleben A, Brudny-Klöppel M, Timmerman P et al. Blood stability testing: European Bioanalysis Forum view on current challenges for regulated bioanalysis. Bioanalysis 3(12), 1333-1336 (2011).

\section{Affiliations}

\section{Nozomu Koseki}

Kyorin Pharmaceutical Co., Ltd., DMPK Research Laboratory, Watarase Research Center, 1848, Nogi, Nogi-machi, Shimotsugagun, Tochigi, 329-0114, Japan

\section{Hiroko Ashizawa}

Kaken Pharmaceutical Co., Ltd., Pharmacokinetics and Safety Department, Drug Research Center, 301 Gensuke, Fujieda-shi, Shizuoka, 426-8646, Japan

\section{Takuho Ishii}

Sunplanet Co., Ltd., DMPK\&Bioanalysis Unit,Tsukuba R\&D Supporting Division, 5-1-3 Tokodai, Tsukuba-shi, Ibaraki, 300-2635, Japan

\section{Takahide Uchimura}

Chugai Research Institute for Medical Science, Inc., 200 Kajiwara, Kamakura, Kanagawa, 247-8530, Japan

\section{Keita Ono}

Taiho Phamaceutical Co., Ltd., Bioanalytical Research \& Development, Pharmacokinetics research Laboratories, Discovery and Preclinical Research Division, 3 Ohkubo, Tsukuba-shi, Ibaraki, 300-2611, Japan

\section{Saori Kuriyama}

Nippon Shinyaku Co., Ltd., Pharmacokinetics and Safety Assessment Department. Discovery Research Laboratories, 14 Nishinoshomonguchi-cho, Kisshoin, Minami-ku, Kyoto, 601-8550, Japan

\section{Akiko Toda}

Shin Nippon Biomedical Laboratories, Ltd., Analysis Research Department, Drug Safety Research Laboratories, 2438 Miyanoura, Kagoshima, 891-1394, Japan
6 Guideline on Bioanalytical Method Validation. European Medicines Agency, London, UK (2011). www.ema.europa.eu/docs/en_GB/document_library/

7 White S, Adcock N, Amsterdam P et al. European Bioanalysis Forum: recommendation for dealing with internal standard variability. Bioanalysis 6(20), 2767-2774 (2014).

8 Lowes S, Hucker R, Jemal M et al. Tiered approaches to chromatographic bioanalytical method performance evaluation: recommendation for best practices and harmonization from the Global Bioanalysis Consortium Harmonization Team. AAPS J. 16(6), 1159-1161 (2015).

9 Timmerman P, Anders Kall M, Gordon B et al. Best practices in a tiered approach to metabolite quantification: views and recommendations of the European Bioanalysis Forum. Bioanalysis 2(7), 1185-1194 (2010).

10 Guideline on Bioanalytical Method Validation in Pharmaceutical Development. Japanese Ministry of Health, Labour and Welfare, Tokyo, Japan (2013). http://bcn2012.europeanbioanalysisforum.eu/slides/

\section{Kuretake Soejima}

Meiji Seika Pharma Co., Ltd., Pharmacokinetics and Analysis Laboratory, Pharmaceutical Research Center, 760 Morooka-cho, Kohoku-ku, Yokohama 222-8567, Japan

\section{Naoko Nakai}

Daiichi Sankyo Co., Ltd., Clinical Pharmacology Department, R\&D Division, 1-2-58 Hiromachi, Shinagawa-ku, Tokyo, 140-8710, Japan

\section{Naohiro Nishimura}

Sumika Chemical Analysis Service, Ltd., Chemicals Compliance Division,22-5 Hongo 3-chome, Bunkyo-ku, Tokyo, 113-0033, Japan

\section{Tsuyoshi Mayumi}

Zensei Pharmaceutical Co., Ltd., Analytical Research \& Development Section, 380 Mita-cho, Kishiwada-shi, Osaka, 596-0808, Japan

\section{Yutaka Yasuda}

Toray Research Center, Inc., Biomedecal Analysis Labolatories, Research Division, 6-10-1 Tebiro, Kamakura, Kanagawa, 248-0036, Japan

\section{Tatsuya Yamakawa}

Fujifilm Corporation, Pharmaceutical \& Healthcare Research Laboratories, Research \& Development Management Headquarters, 2-4-1 Shimookui, Toyama, 930-8508, Japan

\section{Makoto Niwa}

Nippon Kayaku Co., Ltd., Pharmaceutical Research Laboratories, Research \& Development Group, 3-31-12 Shimo, Kita-ku, Tokyo,115-8588, Japan 